\title{
Effective and Structural Connectivity in the Human Auditory Cortex
}

\author{
Jaymin Upadhyay, ${ }^{1,4}$ Andrew Silver, ${ }^{2,3}$ Tracey A. Knaus, ${ }^{2,3}$ Kristen A. Lindgren, ${ }^{2,3}$ Mathieu Ducros, ${ }^{1}$ Dae-Shik Kim, ${ }^{1,2,4}$ \\ and Helen Tager-Flusberg ${ }^{2,3}$ \\ ${ }^{1}$ Center for Biomedical Imaging, ${ }^{2}$ Department of Anatomy and Neurobiology, and ${ }^{3}$ Laboratory of Developmental Cognitive Neuroscience, Department of \\ Anatomy and Neurobiology, Boston University School of Medicine, Boston, Massachusetts 02118, and ${ }^{4}$ Program in Neuroscience, Boston University, \\ Boston, Massachusetts 02215
}

Language processing involves multiple neuronal structures in the human auditory cortex. Although a variety of neuroimaging and mapping techniques have been implemented to better understand language processing at the level of the auditory cortex, much is unknown regarding how and by what pathways these structures interact during essential tasks such as sentence comprehension. In this study, the effective and structural connectivity at the level of the auditory cortex were investigated. First, blood oxygenation leveldependent (BOLD) responses were measured with time-resolved functional magnetic resonance imaging (fMRI) during audition of short sentences. Once BOLD activation maps were obtained, the effective connectivity between primary auditory cortex and the surrounding auditory regions on the supratemporal plane and superior temporal gyrus (STG) were investigated using Granger causality mapping (GCM). Effective connectivity was observed between the primary auditory cortex and (1) the lateral planum polare and anterior STG, and (2) the lateral planum temporale and posterior STG. By using diffusion tensor probabilistic mapping (DTPM), rostral and caudal fiber pathways were detected between regions depicting effective connectivity. The effective and structural connectivity results of the present study provide further insight as to how auditory stimuli (i.e., human language) is processed at the level of the auditory cortex. Furthermore, combining BOLD fMRI-based GCM and DTPM analysis could provide a novel means to study effective and structural connectivity not only in the auditory cortex, but also in other cortical regions.

Key words: auditory cortex; effective connectivity; probabilistic mapping; brain development; diffusion; fMRI

\section{Introduction}

Although the primary auditory cortex (PAC) is known to process properties such as sound frequency, precisely which auditory features are processed in regions rostral and caudal to PAC is not as clear (Formisano et al., 2003; Talavage et al., 2004; Upadhyay et al., 2007). Some report that rostral auditory regions process speech or pitch (Binder et al., 2000; Scott et al., 2000; Hickok and Poeppel, 2004, 2007; Ahveninen et al., 2006; Altmann et al., 2007) and caudal areas are involved with perceiving sound location (Warren et al., 2002; Hickok and Poeppel, 2004, 2007; Zimmer and Macaluso, 2005; Ahveninen et al., 2006; Altmann et al., 2007). These observations are analogous with the rostral "what" and caudal "where" streams in the monkey auditory system (Romanski et al., 1999a,b; Tian et al., 2001). Others report that caudal auditory cortex processes the spectrotemporal features characteristic of sound location and speech. This goes against the presence of strict "what" and "where" streams in human auditory

\footnotetext{
Received May 11, 2007; revised Feb. 11, 2008; accepted Feb. 14, 2008.

This work was supported by a grant from the Nancy Lurie Marks Family Foundation and by the National Institutes of Health (U19 DC 03610). We thank Frank Guenther, Alard Roebroeck, and Itamar Ronen for their assistance throughout this study.

Correspondence should be addressed to Dr. Helen Tager-Flusberg, Department of Anatomy and Neurobiology, 715 Albany Street, L-814, Boston University School of Medicine, Boston, MA 02118. E-mail: htagerf@bu.edu. D01:10.1523/JNEUROSCI.4434-07.2008

Copyright $\odot 2008$ Society for Neuroscience $\quad$ 0270-6474/08/283341-09\$15.00/0
}

cortex (Zatorre et al., 1992; Belin and Zatorre, 2000). It is likely that the caudal circuitry may process both "spectral motion" (speech processing) and "motion of sound" (localization) (Romanski et al., 2000).

Characterizing connectivity can greatly enhance our understanding of how sensory processing is carried out; however, conventional imaging methods such as functional magnetic resonance imaging (fMRI) measure blood oxygenation leveldependent (BOLD) responses but do not have the temporal resolution to resolve the sequence of responses that occur within a circuit. Although the timescale of BOLD signals is not at the same level as neuronal spiking, interactions can be investigated by means of either functional or effective connectivity analysis (Horwitz, 1990, 2003; Friston et al., 1993a,b, 2003; McIntosh and Gonzales-Lima, 1994; McIntosh et al., 1994; Buchel and Friston, 1997; Friston and Buchel, 2000; Friston, 2002). Whereas functional connectivity refers to coherence of activity across the brain, effective connectivity identifies how the activity in one brain region influences another. Previously, Granger causality mapping (GCM) was implemented to identify whole-brain functional and effective connectivity (Granger, 1969, 1980; Goebel et al., 2003; Roebroeck et al., 2005). GCM cannot resolve whether the interaction between two regions is direct or indirect. However, diffusion tensor probabilistic mapping (DTPM) can be used to circumvent this shortcoming, where fibers between regions 
portraying causality are sought (Behrens et al., 2003; Parker et al., 2003).

In this study, effective and structural connectivity in auditory cortex underlying language processing were investigated with GCM and DTPM. Effective connectivity denotes the situation in which the functional activity in region one predicts the functional activity in region two; structural connectivity refers to a white matter pathway between two regions. If the two types of connectivity are characterized, then it may be possible to determine how auditory stimuli similar to human language are propagated in auditory cortex. It was hypothesized that passive listening to short sentences should elicit BOLD responses within auditory cortex. GCM analysis should show effective connectivity between PAC and non-PAC structures and DTPM should reveal whether the connectivity is direct or indirect.

\section{Materials and Methods}

Subjects. Approval for this study was obtained from the Institutional Review Board of Boston University School of Medicine. Data were collected from eight healthy right-handed male subjects between 17 and 25 years old $(20.3 \pm 3.3$ years old). Subjects were native monolingual English speakers. Informed consent was obtained from each volunteer before the scanning session.

$M R$ imaging parameters. All imaging data were collected using a sixchannel SENSE receiver coil on a 3 tesla Philips Intera (Philips Medical Systems, Cleveland, $\mathrm{OH}$ ) scanner housed with $2.2 \mathrm{G} / \mathrm{cm}$ maximum gradient strength. fMRI parameters were as follows: pulse sequence, singleshot spin echo echoplanar imaging (SE-EPI); repetition time (TR)/echo time (TE), $1000 / 28 \mathrm{~ms}$; flip angle, $77^{\circ}$; field of view, $224 \times 224$; imaging resolution, $3.5 \times 3.5 \times 4.0 \mathrm{~mm}$; slice thickness, $4.0 \mathrm{~mm}$ with $1.0 \mathrm{~mm}$ slice gap; number of axial slices, 20. Structural MRI parameters were as follows: pulse sequence, three-dimensional (3D) magnetization-prepared rapid-acquisition gradient echo; TR/TE, 7.47/3.4 ms; flip angle, $8.0^{\circ}$; imaging resolution, $0.9 \times 0.9 \times 1 \mathrm{~mm}$; number of axial slices, $\sim 160$. Diffusion tensor imaging (DTI) parameters were as follows: pulse sequence, single-shot SE-EPI; TR/TE, 10,646/91 ms; $b$ value, $1000 \mathrm{~s} / \mathrm{mm}^{2}$; imaging resolution, $1.8 \times 1.8 \times 2.0 \mathrm{~mm}$; number of diffusion directions, 15 ; number of axial slices, 73 . Three DTI datasets were acquired, motion corrected, coregistered, and averaged within and between acquisitions using Philips software (Philips Medical Systems).

Auditory stimuli. Sentences were spoken by a female and recorded using Adobe Audition 1.5 in a soundproof room (Adobe Systems, San Jose, CA). All stimuli were subsequently normalized for intensity. The presentation of each stimulus was synchronized with the MR scanner using code written in MATLAB 6.5 (MathWorks, Natick, MA) and binaurally presented at a sound intensity of $\sim 70 \mathrm{~dB}$ sound pressure level using standard MRI-compatible pneumatic headphones (Avotec, Stuart, $\mathrm{FL}$ ). The use of the MRI-compatible headphones enabled a sound attenuation of $\sim 20 \mathrm{~dB}$ from the gradient acoustic noise. Thirty-two short and simple sentences (1-2 s in duration) were presented, during functional runs $\sim 6-7 \mathrm{~min}$ long. The interstimulus interval was $10 \mathrm{~s}$. Within a single fMRI run, 384 volumes were continuously collected, where each volume was collected within $1000 \mathrm{~ms}$. Volumes were collected during the start, middle, and end of sentences, as well as during the interstimulus interval. Sentences (e.g." "The woman raked the yard.”) consisted of one- or twosyllable words that were highly concrete, imageable, and familiar. Words were obtained from the Medical Research Council psycholinguistic database (Wilson, 1988). During presentation of auditory stimuli, subjects were asked to be attentive to each stimulus.

fMRI and anatomical data analysis. BrainVoyager QX 1.7.9 software was used for single-subject fMRI and anatomical image analysis (Brain Innovation, Maastricht, The Netherlands). fMRI datasets were preprocessed with a $3 \mathrm{D}$ head motion correction procedure, slice scan time corrected using a sinc interpolation, and high- and low-pass filtered to remove nonlinear drift in the data. Anatomical images were interpolated up to a $1 \times 1 \times 1 \mathrm{~mm}$ isovoxel resolution, transformed into the Talairach coordinate system, and coregistered with each fMRI dataset. Once white matter segmentation of the anatomical datasets was accomplished, the cortical surface was inflated using an automatic brain inflation algorithm. Statistical activation maps were obtained by computing a singlesubject general linear model, where two predictors were defined, one designated for the language stimuli and the other for baseline, where only gradient noise was present. Language stimuli and baseline predictors were each fine tuned with a gamma BOLD response function (onset, $0 \mathrm{~s}$; response and undershoot dispersion, 1; response peak, $5 \mathrm{~s}$ ). To obtain statistical functional maps associated with each language stimulus, the baseline and stimulus predictors were subtracted from each other. The significance of the observed functional maps were jointly assessed using the false discovery rate (FDR), which represents the fraction of false positives in a positive result, and also with a conventional $p$ value (Genovese et al., 2002). The FDR or $q$ value was set to $q=0.01$, while all functional maps assessed were above a significance of $p<0.001$. Lower $q$ values corresponded with lower $p$ values.

Whole-brain GCM analysis. In whole-brain GCM-based effective and functional connectivity analysis, the reference volume of interest (VOI) was considered region $X$, whereas the remaining voxels within the imaging volume $(Y)$ were considered either as possible targets or sources of influence to the reference region. All 384 time points or volumes acquired during the functional run and each voxel within each volume were included in the GCM analysis. Thus, in this study effective connectivity was characterized between the PAC and the entire fMRI volume.

GCM is a vector autoregressive modeling technique used to define effective and/or functional connectivity. As mentioned previously, GCM analysis is optimally implemented on fMRI data collected with TRs or epoch times of $1000 \mathrm{~ms}$ or less (Roebroeck et al., 2005). Here, the fMRI data points collected across time of an individual voxel or averaged time series of voxels within a VOI make up a vector time series, $x_{i}(t)$, where $t$ represents time and $i$ denotes the voxel or VOI. In the case of functional connectivity, a cross-covariance matrix, $C_{x x}(1)$, contains the elements $r_{i i}(1)$, which describe the correlation function between $x_{i}(t)$ and $x_{j}(t)$ at time lag 1 . The element $r_{i i}(1)$ represents the correlation of $x_{i}$ at the same time lag 1 . Thus, the cross-covariance matrix $C_{x x}(1)$ are made up of elements that combine as a function to describe the linear association between time series of voxels or VOIs $i$ and $j$. To identify and characterize effective connectivity in GCM, it is assumed that the vector time series $x_{i}(t)$ is a function of its own past $x_{i}(t-1, \ldots t)$ and possibly $y_{i}(t-1, \ldots t)$. Therefore, it can be stated that if there is causality between $x$ and $y$, the present value of $x$ can be better predicted using the information of both time series $x(t)$ and $y(t)$ compared with solely using the information in time series $x(t)$. Furthermore, the number of time series that can be used to predict the current value $x$ can be more than two.

In GCM analysis, maps were computed from BOLD response time courses, where those regions that were influenced by the reference VOI $(X \rightarrow Y)$ and those regions that influenced the reference VOI $(Y \rightarrow X)$ were identified. As depicted and explained by Roebroeck et al. (2005), biases are intrinsic in Granger causality analysis of fMRI data when solely or independently observing $X \rightarrow Y$ and $Y \rightarrow X$ occurrences; instances of $Y \rightarrow X$ influence occurred at a higher frequency above threshold than $X \rightarrow Y$ interactions. However, by calculating and inferring the direction of influence on the difference between the two types of interactions, $(X \rightarrow Y)-(Y \rightarrow X)$, the initial bias is eliminated. In "difference" GCM, a positive difference value indicates $X \rightarrow Y$ (reference $\rightarrow$ volume) influence, whereas negative difference values depict $Y \rightarrow X$ (volume $\rightarrow$ reference) influence. Moreover, if the BOLD response time courses of $X$ and $Y$ were observed to be correlated and no direction of influence was present, instantaneous causality among $X$ and $Y$ was present, thus representing functional connectivity. More specifically, if inclusion of a current value $X$ improved the prediction of a current $Y$ value at the same time point, functional connectivity existed between $X$ and $Y$. Statistical significance thresholds for the functional and effective connectivity maps were computed by bootstrapping and applying a conservative FDR value of at least $q=0.01$ for a multiple comparison correction (Genovese et al., 2002). Activation in PAC (approximately the posterior half of Heschl's gyrus (HG), $\sim 400$ voxels in the $\mathrm{T}_{1}$-weighted anatomical dataset) was defined as the initial reference VOIs and used during GCM analysis to identify effective connectivity in the auditory cortex. To define the PAC on Hes- 


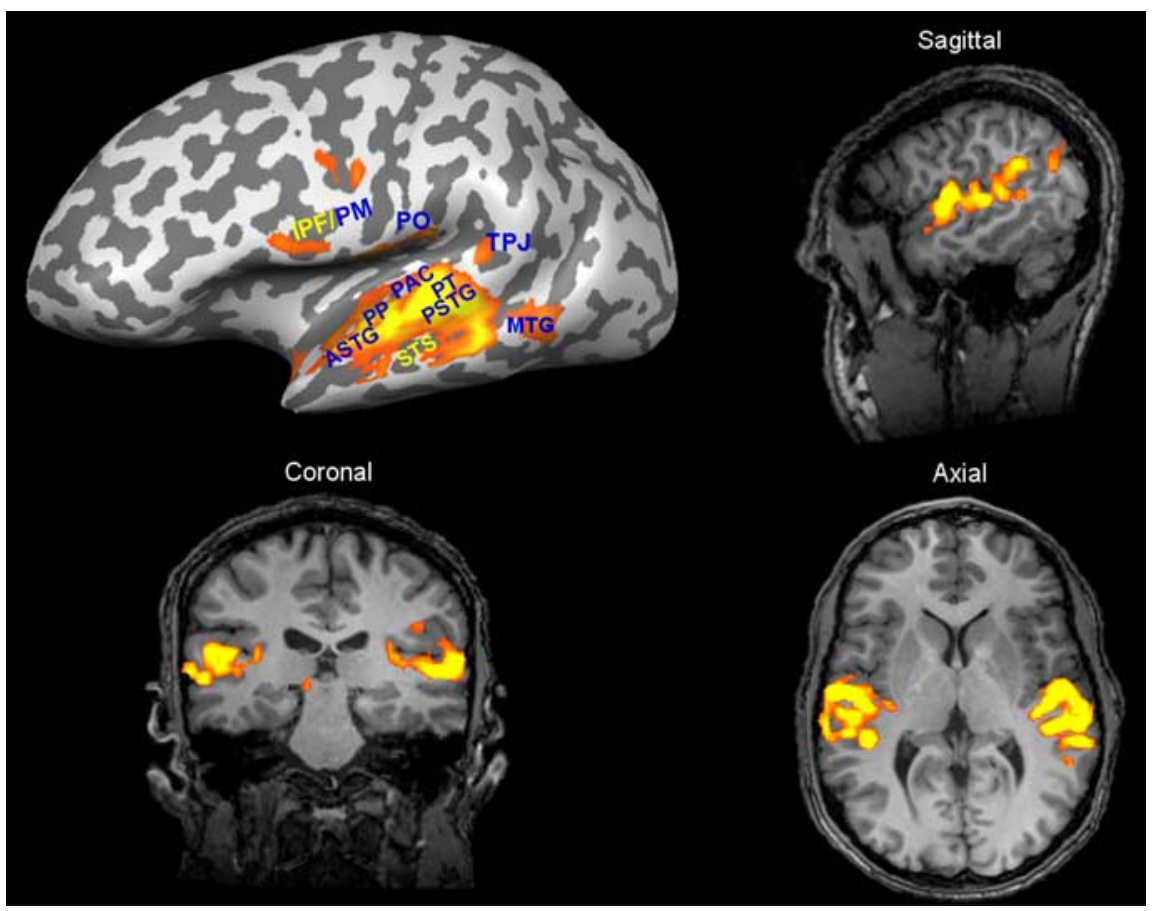

Figure 1. Cortical BOLD activation during sentences processing. In all subjects, activation was present in the left and right hemispheres when passively listening to sentences. BOLD activation was strongest and most consistent across all subjects in the left hemisphere auditory cortex. Significant activity not only occurred in superior temporal regions, but also in other auditory or language relevant areas. All BOLD activation maps were produced with the same threshold or significance level $(p<0.001)$.PP, Planum polare; MTG, middle temporal gyrus; $\mathrm{PO}$, parietal operculum; TPJ, temporal parietal junction; $\mathrm{PM}$, premotor cortex; IPF, inferior prefrontal cortex.

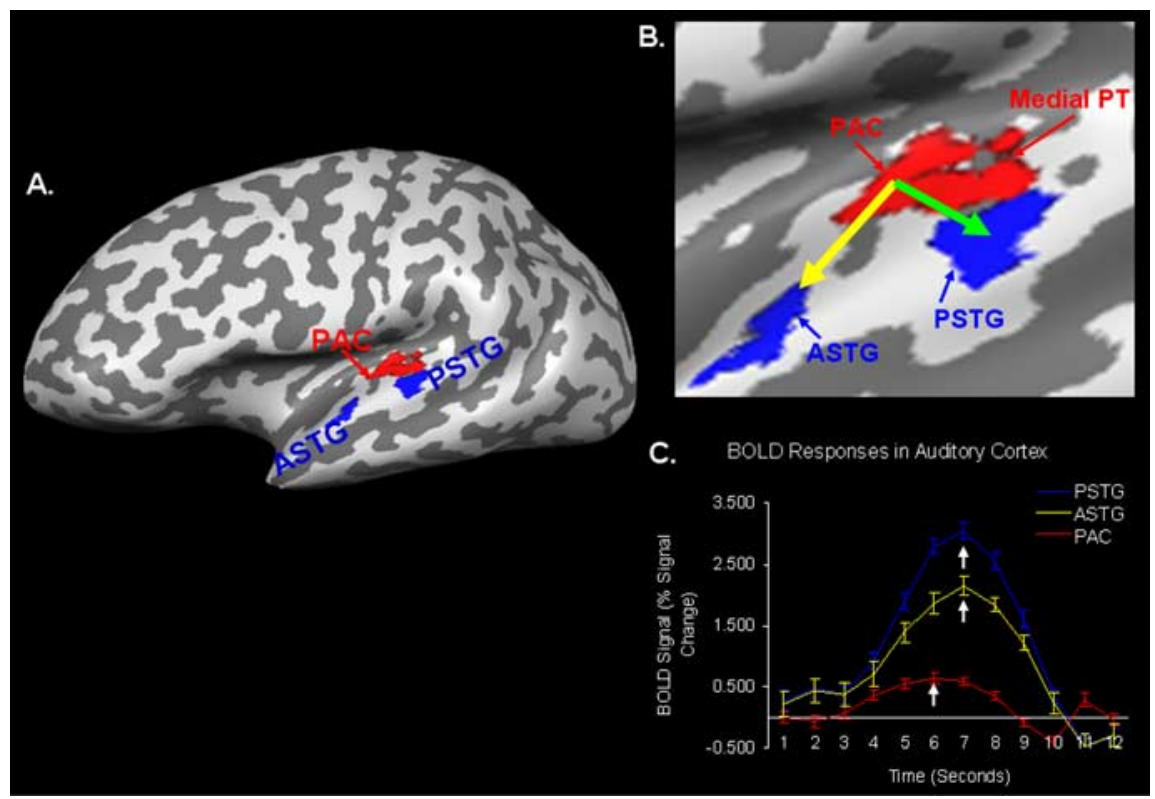

Figure 2. Effective connectivity in auditory cortex I.A, An effective connectivity map in the auditory cortex is shown for a single subject. The red region corresponds to the reference region in PAC as well as the medial regions of the PT, which showed functional connectivity with PAC. Blue regions depict the rostrolateral (lateral planum polare + anterior STG; referred to as ASTG) and caudolateral (lateral planum temporale + posterior STG; referred to as PSTG) auditory cortex regions identified as being targets of influence of PAC. $B$, An enlarged view of the effective connectivity map is shown on the inflated brain surface. The green arrow depicts a caudal pathway as defined by GCM analysis, and the yellow arrow represents a possible rostral pathway. $\boldsymbol{C}$, The averaged BOLD response time courses for the PAC and rostrolateral and caudolateral clusters are given. White arrows indicate temporal points at which the peak in the BOLD response occurred. GCM results using more localized VOls in Heschl's gyrus are shown in supplemental Figure 2 (available at www.jneurosci.org as supplemental material), whereas all single-subject $(n=8)$ effective connectivity maps are shown in supplemental Figure 3 (available at www.jneurosci.org as supplemental material). chl's gyrus, we used a combination of past anatomical work by Hackett et al. (2001), Morosan et al. (2001), and Rademacher et al. (2001), in conjunction with results from intracranial recording studies in humans by Howard et al. (2000) and Brugge et al. (2003). Furthermore, the transverse temporal sulcus was used to define the border between Heschl's gyrus and planum temporale (PT), whereas the first temporal sulcus was used to define the border between Heschl's gyrus and planum polare.

DTI and probabilistic mapping analyses. Single-subject DTI and probabilistic mapping analyses were performed using an in-house MATLAB-based software package (MathWorks) (Lehericy et al., 2004; Upadhyay et al., 2007). DTI and anatomical datasets were first manually coregistered using the corpus callosum as well as the right and left lateral sulci as landmarks. EPI-based statistical maps were created in the BrainVoyager QX 1.7.9 environment, where maps represented the BOLD responses elicited during sentence processing. Statistical maps along with the respective echoplanar images were then coregistered with the combined non-Talairach DTI and anatomical dataset. This enabled VOIs to be created in the combined DTI and anatomical datasets where VOIs represented activated neuronal structures, and also the neuronal structures defined as having effective connectivity among each other as defined by GCM.

A PAC VOI in the coregistered DTI dataset was first defined both functionally and anatomically. A good majority, but not all, of the regions of Heschl's gyrus were functionally active or portrayed effective connectivity with other regions of the auditory cortex. However, to identify any and all possible fiber projections between the PAC and those regions showing effective connectivity with it, the entire posterior half of Heschl's gyrus was marked as the VOI used during probabilistic mapping. Two of the eight subjects possessed a rostral and caudal Heschl's gyrus. In these cases, two VOI were made, one consisting of both gyri and one VOI consisting of only the rostral gyrus, which is considered to correspond to the true PAC (Sigalovsky et al., 2006). The caudal Heschl's gyrus is thought to be an extension of the planum temporale. Other non-PAC regions were defined solely based on functional activity and Granger causality maps. Each VOI was identified on a slice by slice basis and initially confined to gray matter. Therefore, it was necessary to extend VOIs to include $\sim 3-4 \mathrm{~mm}$ of adjacent white matter, enabling probabilistic mapping to be performed (supplemental Fig. 1, available at www.jneurosci.org as supplemental material).

Probabilistic mapping was achieved with the Monte Carlo probabilistic mapping method proposed by Parker et al. (2003) and described in great detail by Upadhyay et al. (2007). In this probabilistic mapping method, 1000 fibers were generated from each seeding voxel. The probability value, $P$, for each voxel containing axonal projections was obtained with the equation $P=$ $100 \times N / N_{\text {fibers }}$ (in percentage), where $N$ is the number of fibers going through a given voxel in 


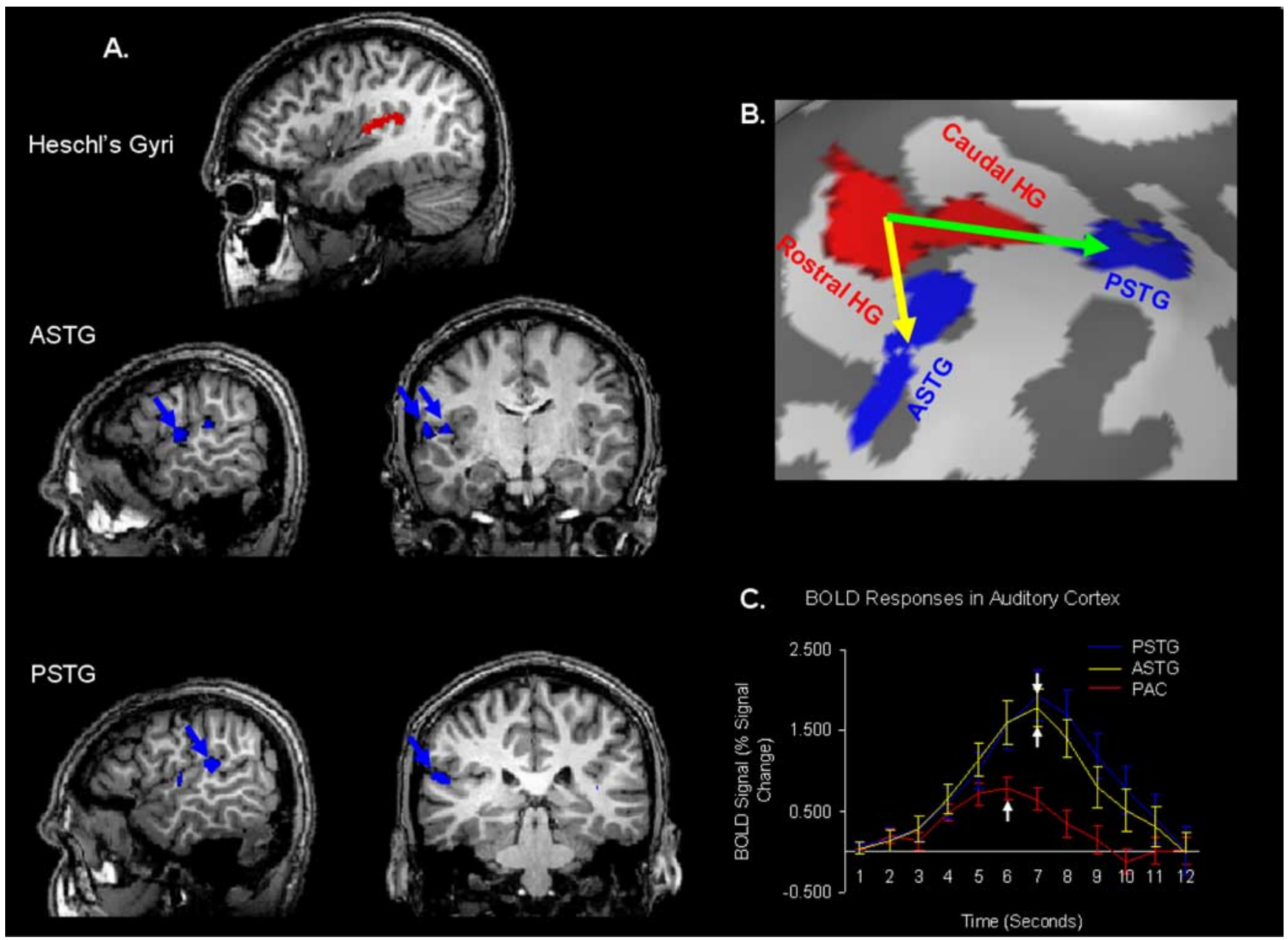

Figure 3. Effective connectivity in auditory cortex II. A, Effective connectivity maps are shown for a subject with a rostral and caudal Heschl's gyrus. In the top row, the rostral and caudal Heschl's gyri are marked in red. On the left side, the rostrolateral and caudolateral clusters identified by GCM analysis are shown on $\mathrm{T}_{1}$-weighted sagittal slices, whereas on the right these maps are shown in the coronal view. B, Effective connectivity maps are shown on the inflated brain surface. Functional connectivity was observed between rostral and caudal Heschl's gyri. The green arrow depicts a caudal pathway as defined by GCM analysis, whereas the yellow arrow represents a possible rostral pathway. $C$, The averaged BOLD response time courses for the PAC and rostrolateral and caudolateral clusters are shown. White arrows indicate temporal points at which the peak in the BOLD response occurred in each of the three time course. All single-subject ( $n=8)$ effective connectivity maps are shown in supplemental Figure 3 (available at www.jneurosci.org as supplemental material).

the fiber bundle and $N_{\text {fibers }}$ is the total number of fibers connecting both VOIs. Many fibers that are created in the Monte Carlo simulations do not reach both of the target VOIs. These latter fibers are not taken into account in the calculation of the probability, $P$. Probability maps are normalized to the total number of fibers, $N_{\text {fibers }}$. Probabilistic mapping was limited to fiber projections among two regions that showed effective connectivity. Thus, the probability maps computed represent the DTIbased fiber density of the bundle between the regions or VOIs showing effective connectivity and an indication of the most likely path between the two VOIs. A VOI-to-VOI probabilistic mapping method significantly reduces the creation of false fibers as a result of the anatomical constraints imposed, and also allows for a characterization and quantification of a particular segmented fiber pathway. In addition to the segmentation of fiber pathways, probabilistic mapping enabled a quantification of the probability (fiber density) for each fiber bundle.

\section{Results}

Significant $(p<0.001)$ BOLD responses in the left hemisphere auditory cortex during audition of sentences were present in all subjects (Fig. 1). Activity was also seen in other cortical areas, such as inferior prefrontal cortex and superior temporal sulcus (STS). However, across all subjects the strongest and most consistent activity was observed in the left hemisphere auditory cor- tex [supratemporal plane + superior temporal gyrus (STG)]. In Figure 1, the inflated brain surface and 2D slices show significant and strong BOLD activation during sentence processing in auditory structures including PAC (Heschl's gyrus), posterior STG, and anterior STG.

Ipsilateral effective connectivity was investigated with GCM analysis and was performed using functional activation maps acquired during sentence processing in the left hemisphere auditory cortex. As shown in Figure 2, GCM revealed significant ( $q=$ 0.01 ) effective connectivity between PAC and two lateral regions near the border of lateral supratemporal plane and STG. One lateral region or cluster was located slightly rostral to PAC and on the border of lateral planum polare and anterior STG (ASTG). The more rostrolateral region is collectively referred to as ASTG. The second region identified by GCM was caudal to PAC and on the border of lateral planum temporale and posterior STG (PSTG). This caudolateral region is collectively referred to as PSTG. In Figure $2 A$, the effective connectivity maps are shown on the inflated brain surface for a single subject. An enlarged view of this connectivity map on the inflated brain surface is presented in Figure $2 B$. The red cluster indicates PAC (reference region), 
whereas the blue clusters indicate ASTG and PSTG. Although the original reference region only included the PAC on Heschl's gyrus, the red reference region was expanded and showed significant functional connectivity with the very medial regions of the planum temporale [the following color coding scheme was used in all GCM maps shown: (1) red, reference VOI and regions showing functional connectivity; and (2) blue, possible targets of influence of the reference VOI or activation occurring after reference VOI activation]. In Figure $2 C$, the BOLD response time courses are given for each of the three clusters. It can be seen that the peak in the BOLD response for the PAC cluster occurs approximately $1 \mathrm{~s}$ before the BOLD response peaks of the ASTG and PSTG clusters. Furthermore, the ASTG and PSTG BOLD responses peak at approximately the same time point.

The reference VOI in Figure 2 expanded across Heschl's gyrus and likely included primary and primary-like auditory fields along the gyrus, where each subfield may have slightly different effective connectivity. To address this issue, two smaller reference VOIs were positioned in Heschl's gyrus, and GCM mapping was performed (supplemental Fig. 2, available at www.jneurosci.org as supplemental material). One VOI was medially located in $\mathrm{He}-$ schl's gyrus, whereas the other was adjacent and caudomedial. This latter GCM procedure yielded a result such that similar effective connectivity maps were observed between those initially observed in Figure 2 (same data shown in supplemental Fig. $2 \mathrm{~A}$, available at www.jneurosci.org as supplemental material) and maps depicted in supplemental Figure $2 B$ (available at www.jneurosci.org as supplemental material). In the middle and right panels in supplemental Figure $2 B$, two lateral clusters, ASTG and PSTG, possessing effective connectivity with the medial and caudomedial reference regions are shown. The subsequent GCM procedure did not produce identical effective connectivity maps as depicted in Figure 2. Nonetheless, the principle observation of anterior and posterior clusters along STG having effective connectivity with the medial half of Heschl's gyrus held true.

Figure 3 shows effective connectivity maps in which the subject had both a rostral and caudal Heschl's gyrus on $2 \mathrm{D} \mathrm{T}_{1}$ weighted images (Fig. $3 A$ ) and on the inflated brain representation (Fig. $3 B$ ). The effective connectivity maps and temporal properties of the BOLD responses of each cluster (Fig. $3 C$ ) were similar compared with those effective maps of subjects with a single Heschl's gyrus. The GCM analysis showed effective connectivity between Heschl's gyri and ASTG and also between Heschl's gyri and PSTG. Functional connectivity was observed between the rostral and caudal Heschl's gyri.

Effective connectivity patterns shown in Figures 2 and 3 were consistently observed across all subjects. Supplemental Figure 3 (available at www.jneurosci.org as supplemental material) shows effective connectivity maps in left hemisphere auditory cortex for all eight subjects; VOI-constrained DTPM was performed to validate these effective connectivity maps, and to decipher whether the regions showing effective connectivity are structurally connected by direct or indirect means. As mentioned above, the effective connectivity maps did not show a clear distinction between the lateral planum polare and lateral planum temporale from ASTG and PSTG, respectively. Thus, in DTPM analysis, the lateral planum polare + ASTG, as identified and defined by GCM analysis, was considered as a single cluster or seeding VOI, as was the lateral planum temporale + PSTG. Figure 4 shows two fiber pathways in the auditory cortex for a subject with two Heschl's gyri, a rostral pathway and a caudal pathway. The rostral pathway (1) emanates from the rostral regions of the rostral Heschl's gyrus, (2) projects rostrally and laterally toward the temporal pole,

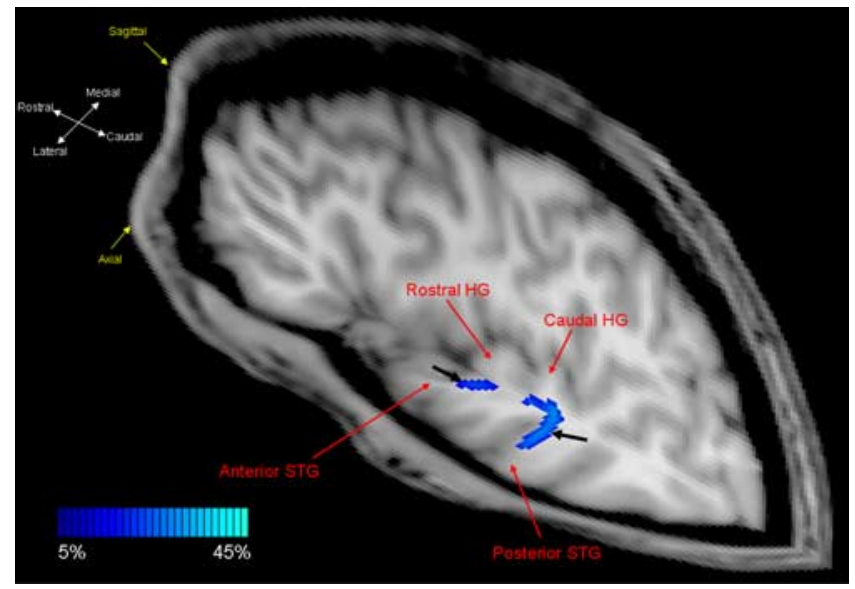

Figure 4. Rostral and caudal fiber pathways in auditory cortex. Two-dimensional probabilistic maps of the rostral and caudal fiber pathways (marked by the black arrows) are shown on sagittal and axial $\mathrm{T}_{1}$-weighted slices. Whereas the caudal pathway projects from more caudal regions of the rostral Heschl's gyrus, the rostral pathway projects from the rostral segments of the same gyrus. The two pathways also have distinct fiber densities or probabilities as defined by probability values (Table 1). High fiber density is indicated by light blue-green voxels; low fiber density is indicated by dark blue voxels.

and (3) terminates in the ASTG. The caudal pathway (1) emanates from caudal regions of the rostral Heschl's gyrus, (2) projects to the caudal Heschl's gyrus, (3) projects laterally from the caudal Heschl's gyrus, and (4) terminates in the PSTG. Compared with the rostral pathway, the caudal pathway consistently had a higher probability or density as defined by probabilistic mapping for each subject (Table 1).

Two-dimensional probabilistic maps of the rostral fiber pathways of the auditory cortex not only had a lower probability, but also traversed a slightly shorter distance. Figures 4 and 5 show similar rostral fiber pathways across four subjects, which did not differ whether one or two Heschl's gyri were present. In the sagittal planes, regions of the rostral pathway closer to the termination point in rostral Heschl's gyrus is shown, whereas in the axial planes the termination point closer to anterior STG is shown.

Two-dimensional probabilistic maps of the caudal fiber pathway on the axial and coronal planes for three subjects are shown in Figure 6. These are the same subjects as those shown in Figure 5 . In the top and bottom rows of Figure 6, it can be seen that subjects with a single Heschl's gyrus showed a more direct pathway projecting between caudal regions of Heschl's gyrus and PSTG. In Figure 6, the middle set of axial and coronal images correspond to a second subject with two Heschl's gyri. Here, it can be seen that this subject had a similar caudal trajectory to that shown in Figure 4 and also in supplemental Figure 4 (available at www.jneurosci.org as supplemental material). It is noted that the caudal fiber projections, either in the single or double Heschl's gyrus case, did not project from the extreme caudomedial plane of Heschl's, but were slightly more lateral. Furthermore, in subjects with two Heschl's gyri, the caudal pathway showed an identical trajectory when either both Heschl's gyri or only the rostral Heschl's gyrus was used as a seeding VOI.

\section{Discussion}

fMRI-based GCM and DTPM were used to investigate the connectivity that underlies language processing in human auditory cortex. Across subjects, significant and consistent BOLD responses were detected in the left hemisphere auditory cortex (Fig. 1). Subsequently, GCM was used to identify effective connectivity 
Table 1. Probability values of caudal and rostral fiber pathways in auditory cortex

\begin{tabular}{|c|c|c|c|c|c|c|c|c|}
\hline & Subj. 1 & Subj. 2 & Subj. 3 & Subj. 4 & Subj. 5 & Subj. 6 & Subj. 7 & Subj. 8 \\
\hline Rostral pathway & 12.86 & 19.91 & 12.93 & 11.99 & 10.15 & 14.15 & 13.18 & 14.54 \\
\hline Caudal pathway & 20.64 & 22.45 & 22.42 & 19.52 & 16.37 & 16.93 & 19.41 & 16.16 \\
\hline
\end{tabular}

Single-subject probability values are given for the caudal and rostral pathways. The caudal pathway consistently showed a higher probability than the rostral pathway across all subjects. The mean \pm SD for the rostral and caudal pathways was $13.71 \pm 2.84$ and $19.24 \pm 2.55$, respectively $(p<0.001)$. Subj., Subject.

between PAC and rostrolateral and caudolateral regions of auditory cortex. For subjects with a single Heschl's gyrus, functional connectivity was observed between Heschl's gyrus and medial regions of the planum temporale (Fig. 2). For subjects with both rostral and caudal Heschl's gyri, functional connectivity was seen between the two (Fig. 3). This similarity in functional connectivity across the two variations in auditory cortex is not surprising given that the more rostral gyrus is considered to be the PAC (Sigalovsky et al., 2006), whereas the caudal gyrus can be considered an extension of planum temporale. DTPM showed that regions depicting effective connectivity were structurally connected (Figs. 4-6). A caudal fiber pathway projecting between caudal regions of Heschl's gyrus and PSTG was observed (Fig. 6), whereas the rostral pathway had a shorter trajectory spanning rostral regions of Heschl's gyrus to ASTG (Fig. 5). The DTPM analysis also showed that the rostral pathway had a lower probability or density compared with the caudal pathway (Table 1).

Based on GCM analysis, the medial regions of the auditory cortex showed effec-

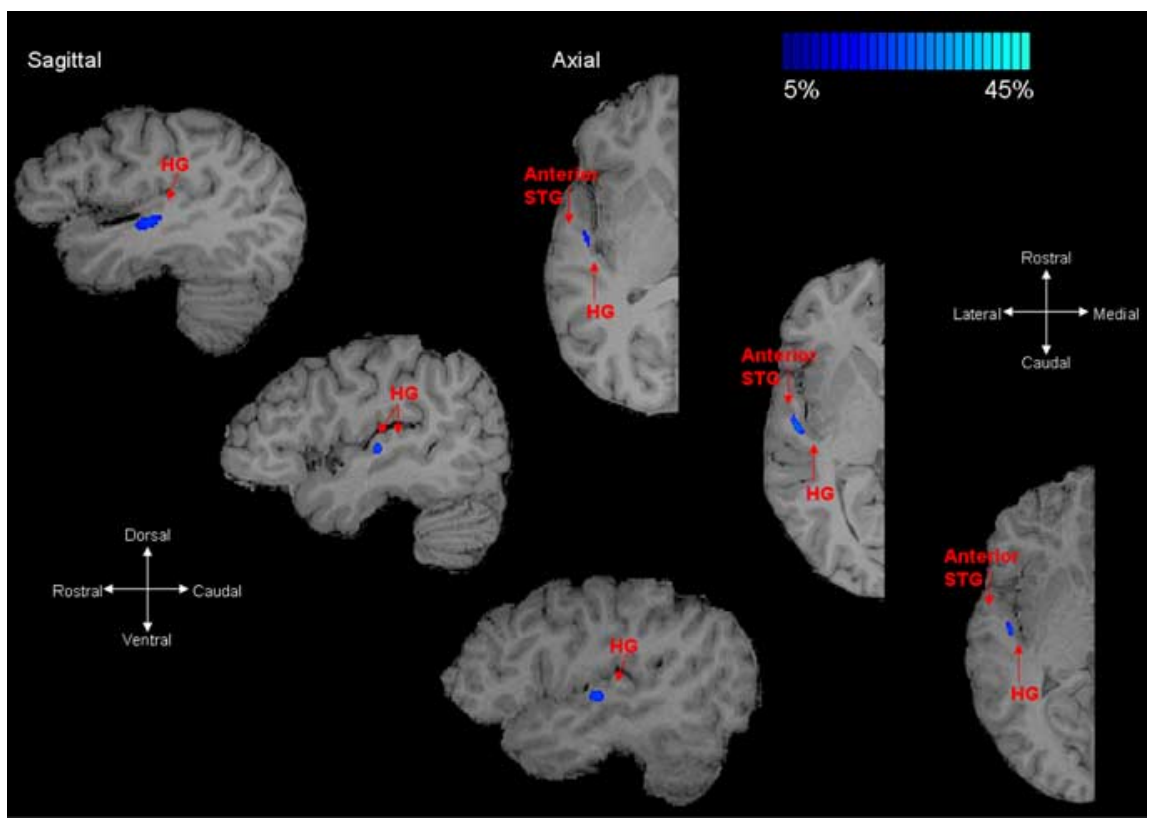

Figure 5. Rostral fiber pathways in auditory cortex. Two-dimensional probabilistic maps of the rostral auditory cortex pathways for three different subjects are shown on axial and coronal $\mathrm{T}_{1}$-weighted slices. This rostral pathway projects between the rostral regions of Heschl's gyrus toward lateral regions of the planum polare and anterior STG. In the sagittal planes, the regions of the rostral pathway closer to the Heschl's gyrus are shown, whereas in the axial view, segments of pathway close to the lateral planum polare-anterior STG border are shown. A difference in fiber trajectories between subjects with one or two Heschl's gyri was not observed. Single-subject and group averaged probability values for the rostral fiber pathways are given in Table 1. High fiber density is indicated by light blue-green voxels; low fiber density is indicated by dark blue voxels.

tive connectivity with lateral auditory cor-

tex (lateral planum temporale + PSTG). Thus, the lateral auditory areas were identified as being downstream to medial auditory cortex. A distinction was not observed among the lateral regions on the supratemporal plane (lateral plana temporale and polare) with anterior and posterior STG. Nonetheless, effective connectivity along rostrolateral and caudolateral orientations in the auditory cortex was observed, which likely reflects the dual processing streams observed in studies of humans (Alain et al., 2001; Ahveninen et al., 2006) and monkeys (Tian et al., 2001). The effective connectivity maps could also reflect that an analogous hierarchical processing stream exists in human auditory cortex comparable with the core, belt, and parabelt auditory fields in nonhuman primates (Pandya, 1995; Kaas and Hackett, 2000). If three distinct hierarchical regions in the human auditory cortex exist, as they do in other primates, it is possible that the difference in the BOLD response onsets between adjacent auditory fields occur at time intervals less than $1 \mathrm{~s}$, which would require fMRI data collection with a TR of less than $1 \mathrm{~s}$ to further resolve the effective connectivity.

The ipsilateral effective connectivity between PAC and PSTG can best be explained by relating these observations with past intracranial recording and anatomical tracer studies performed in humans and monkeys. Howard and colleagues (Howard et al., 2000; Brugge et al., 2003) observed that stimulation of the medial half of Heschl's gyrus yielded the highest activity recorded in ipsilateral posterior STG, demonstrating that medial portions of Heschl's gyrus and posterior STG are components of the same neuronal circuitry. Based on their observations and the intracranial recordings of Liegeois-Chauvel et al. (1991, 1994), it was argued that the planum temporale was a likely intermediary point between medial Heschl's gyrus and posterior STG. Tracer studies performed in nonhuman PAC identified axonal projections between the core and belt regions and between the belt and parabelt auditory cortices (Hackett et al., 1998; Kaas and Hackett, 2000). Furthermore, Tardif and Clarke (2001) demonstrated the presence of short projections within Heschl's gyrus and longer projections emanating from planum temporale and polare. The results of several neuroimaging studies in humans point to a hierarchical processing stream between Heschl's gyrus and rostrolateral auditory cortex (Griffiths et al., 1998; Binder et al., 2000; Scott et al., 2000; Alain et al., 2001; Hickok and Poeppel, 2004, 2007; Ahveninen et al., 2006).

Effective connectivity maps provided a strong indication of rostral and caudal pathways in the auditory cortex. However, two questions remain. Is the connectivity between medial and lateral auditory cortex structures direct or indirect? Do the rostrolateral and caudolateral effective connectivity pathways originate from the same regions of PAC? The entire length of PAC on Heschl's gyrus was used as a seeding VOI for the DTPM analysis, showing that the rostral fiber pathway was a short and direct pathway projecting between rostrolateral Heschl's gyrus and the ASTG 


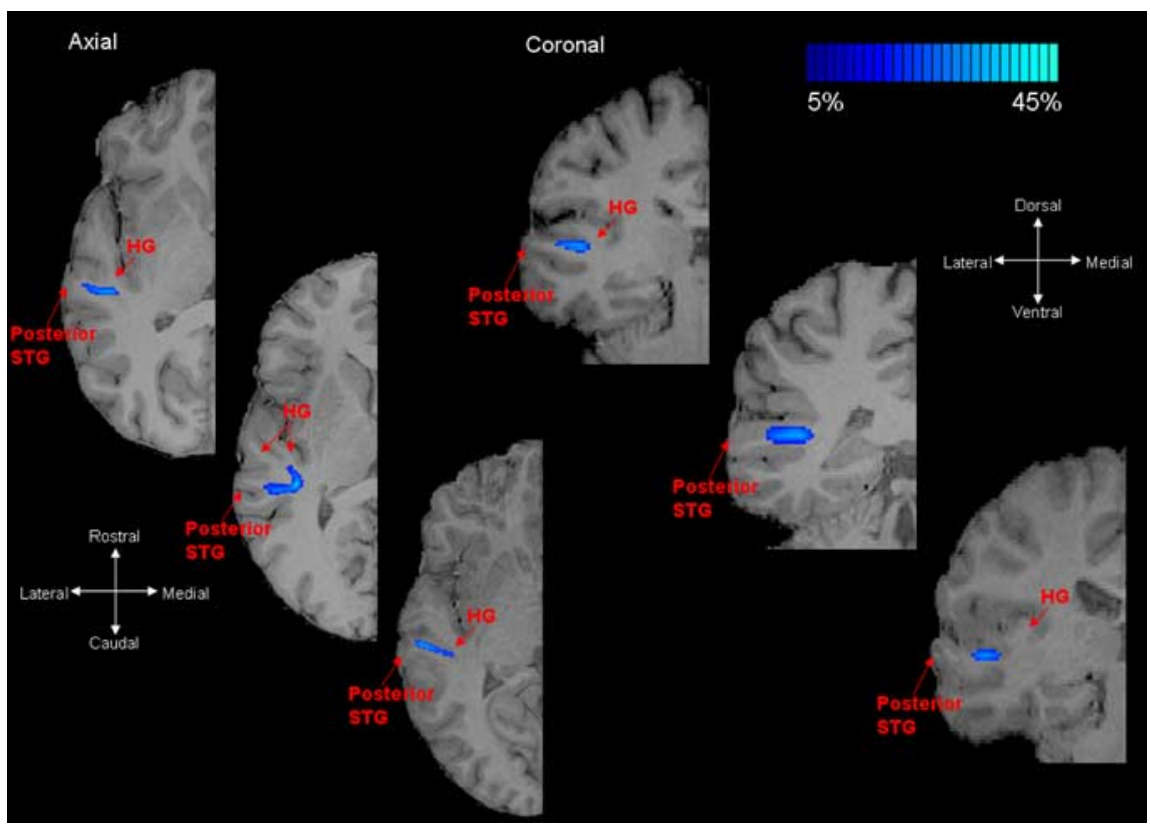

Figure 6. Caudal fiber pathways in auditory cortex. Two-dimensional probabilistic maps of the caudal auditory cortex pathways for three different subjects are shown on axial and coronal $\mathrm{T}_{1}$-weighted slices. The three subjects correspond to the same subjects shown in Figure 5 and are also given in the same order. This pathway principally projects between the caudal regions of Heschl's gyrus to posterior STG. As can be clearly seen in the axial plane, for subjects with two Heschl's gyri, the caudal pathway differed slightly. In the latter case, the caudal trajectory projected toward the caudal Heschl's gyrus/planum temporale and then projected laterally to STG. A comparison between two subjects with two Heschl's gyri is given in supplemental Figure 4 (available at www.jneurosci.org as supplemental material). Single-subject and group averaged probability values for the caudal fiber pathways are given in Table 1. High fiber density is indicated by light blue-green voxels; low fiber density is indicated by dark blue voxels.

cluster. This pathway showed a low but substantial probability or density, which may be because this particular fiber pathway was short. The caudal pathway projected between more caudomedial sections of Heschl's gyrus and the PSTG cluster. Whether subjects had one or two Heschl's gyri, the region of Heschl's gyrus from which the caudolateral pathway projected was not the extreme caudomedial plane of the gyrus. For subjects with both a rostral and caudal Heschl's gyrus, the caudal projection started from the rostral Heschl's gyrus and the pathway traversed the white matter regions underlying the rostral half of planum temporale. The rostral supratemporal area lying caudal to Heschl's gyrus corresponded well with medial planum temporale, which showed functional connectivity with PAC, and lateral planum temporale, which showed effective connectivity along with posterior STG. The caudal pathway showed a high probability or density compared with the rostrolateral pathway, which could be because of the longer distance this specific pathway traverses. Although the trajectory patterns of axons of the auditory cortex have been characterized, a concise histological account of biophysical properties such as axonal diameter or axonal density has yet to be determined. Following Tardif and Clarke (2001), the caudal fiber pathway identified here is likely to be a combination of the PAC to planum temporale and planum temporale to posterior STG pathways, which is supported by the finding that in subjects with two Heschl's gyri this pathway has a caudal projection before a lateral one.

The rostral and caudal pathways observed in this study agree with structural connectivity work in monkey auditory cortex (Galaburda and Pandya, 1983; Morel and Kaas, 1992; Pandya, 1995; Hackett et al., 1998; Kaas and Hackett, 2000). The axons in monkey auditory cortex are known to project between the pri- mary core, belt, and parabelt auditory fields. Furthermore, the caudal pathways originate in the caudal core and project primarily in the caudal belt regions, which in turn project to caudal parabelt regions. An analogous connectivity pattern holds true for the rostral pathways in monkey auditory cortex. Connectivity between caudal and rostral auditory regions in nonhuman primates has been shown to exist, but to a lesser extent than the caudal-to-caudal or rostral-to-rostral connectivity. Although the GCM and DTPM analysis did not reveal this exact connectivity pathway among three distinct hierarchical organized auditory fields, the effective and structural connectivity pathway in the human auditory cortex appears to be similarly organized. Last, the monkey auditory core contains three primary and primary-like auditory fields (A1, R, and RT), where each core region projects to adjacent belt and parabelt regions. This would suggest that human auditory cortex should also consist of at least three distinct processing streams, although only two streams were detected in the present study, perhaps because we only used a passive language listening task, as well as in the earlier studies mentioned above.

The present study is the first to characterize whole-brain functional and effective connectivity of auditory fMRI data using GCM analysis. Recent work by Griffiths et al. (2007) and Kumar et al. (2007) applied dynamic causal modeling to investigate the effective connectivity within the temporal cortex during spectral envelope analysis of synthesized auditory stimuli. In these studies, a serial processing stream between HG and PT and between PT and STS was detected. Griffiths et al. (2007) suggested that the same serial processing stream, HG-PT-STS, could explain deficits in auditory processing observed in a patient suffering from dystimbria. In our study, the serial streams between PT and STS and between planum polare and STS were not observed in the current GCM analysis, nor was GCM analysis performed where planum temporale or polare was used as a reference VOI during GCM analysis. The latter analysis may have yielded serial circuitry described by Griffiths et al. (2007) (i.e., PT to STS). These contrasting findings may be related to differences in the types of auditory stimuli presented. Our results do suggest parallel processing streams between PAC and anterior auditory cortex and between PAC and posterior auditory cortex. We note that it is likely that the auditory processing stream is a hybrid system consisting of both serial and parallel circuitry, and which type of circuitry is dominant could in part be determined by the type of auditory stimuli presented and tasks performed.

\section{Study limitation}

Although the results of this study were obtained using language stimuli, it is likely that the results of this work also reflect the neural substrates, effective connectivity, and structural connectivity necessary for lower-level auditory processing. A variety of auditory stimuli share acoustic properties similar to those present in language stimuli; the same auditory cortex processing stream 
may support a diverse range of auditory stimuli. In the present study, functional activation maps were observed in the left and right hemisphere auditory cortex. However, across the eight subjects, the functional maps and effective connectivity patterns were only consistent in the left hemisphere. For example, in some subjects the activation in the anterior superior temporal gyrus and supratemporal plane was less prevalent and robust compared with posterior regions, which is not surprising considering that language processes are left hemisphere lateralized (Geschwind and Levitsky, 1968). Given the inconsistency observed across subjects, it was not possible to make conclusions about connectivity patterns in both left and right auditory cortices.

\section{Conclusions}

The results of this study provide further evidence that there are at least two processing streams in the human auditory cortex. Whether or not the dual processing streams correspond to the "what" and/or "where" pathway cannot be determined; however, we can infer the means by which information is propagated in the human auditory cortex. The combination of implementing GCM with DTPM provided a unique way to examine effective and structural connectivity. It would be interesting to extend this technique to other experimental paradigms. For example, if the speech-motor circuitry or neuronal substrates could be elicited during speech production, would a third processing stream be detected involving more caudal regions of planum temporale, or is this region integrated only with nonprimary auditory substrates (Hickok and Poeppel, 2004; Guenther et al., 2006)?

\section{References}

Ahveninen J, Jaaskelainen IP, Raij T, Bonmassar G, Devore S, Hamalainen M, Levanen S, Lin FH, Sams M, Shinn-Cunningham BG, Witzel T, Belliveau JW (2006) Task-modulated "what" and "where" pathways in human auditory cortex. Proc Natl Acad Sci USA 103:14608-14613.

Alain C, Arnott SR, Hevenor S, Graham S, Grady CL (2001) "What" and "where" in the human auditory system. Proc Natl Acad Sci USA 98:12301-12306.

Altmann CF, Bledowski C, Wibral M, Kaiser J (2007) Processing of location and pattern changes of natural sounds in the human auditory cortex. NeuroImage.

Behrens TEJ, Johansen-Berg H, Woolrich MW, Smith SM, WheelerKingshott CAM, Boulby PA, Barker GJ, Sillery EL, Sheehan K, Cicarelli O, Thompson AJ, Brady JM, Matthews PM (2003) Noninvasive mapping of connections between human thalamus and cortex using diffusion imaging. Nat Neurosci 6:750-757.

Belin P, Zatorre RJ (2000) "What", "where" and "how" in auditory cortex. Nat Neurosci 3:965-966.

Binder JR, Frost JA, Hammeke TA, Bellgowan PS, Springer JA, Kaufman JN, Possing ET (2000) Human temporal lobe activation by speech and nonspeech sounds. Cereb Cortex 10:512-528.

Brugge JF, Volkov IO, Garell PC, Reale RA, Howard III MA (2003) Functional connections between auditory cortex on Heschl's gyrus and on the lateral superior temporal gyrus in humans. J Neurophysiol 90:3750-3763.

Buchel C, Friston KJ (1997) Modulation of connectivity in visual pathways by attention: cortical interactions evaluated with structural equation modelling and fMRI. Cereb Cortex 7:768-778.

Formisano E, Kim DS, Di Salle F, van de Moortele PF, Ugurbil K, Goebel R (2003) Mirror-symmetric tonotopic maps in human primary auditory cortex. Neuron 40:859-869.

Friston K (2002) Beyond phrenology: what can neuroimaging tell us about distributed circuitry? Annu Rev Neurosci 25:221-250.

Friston KJ, Buchel C (2000) Attentional modulation of effective connectivity from V2 to V5/MT in humans. Proc Natl Acad Sci USA 97:7591-7596.

Friston KJ, Frith CD, Liddle PF, Frackowiak RS (1993a) Functional connectivity: the principal-component analysis of large (PET) data sets. J Cereb Blood Flow Metab 13:5-14.

Friston KJ, Frith CD, Frackowiak RS (1993b) Principal component analysis learning algorithms: a neurobiological analysis. Proc Biol Sci 254:47-54.
Friston KJ, Harrison L, Penny W (2003) Dynamic causal modelling. NeuroImage 19:1273-1302.

Galaburda AM, Pandya DN (1983) The intrinsic architectonic and connectional organization of the superior temporal region of the rhesus monkey. J Comp Neurol 221:169-184.

Genovese CR, Lazar NA, Nichols T (2002) Thresholding of statistical maps in functional neuroimaging using the false discovery rate. NeuroImage 15:870-878.

Geschwind N, Levitsky W (1968) Human brain: left-right asymmetries in temporal speech region. Science 161:186-187.

Goebel R, Roebroeck A, Kim DS, Formisano E (2003) Investigating directed cortical interactions in time-resolved fMRI data using vector autoregressive modeling and Granger causality mapping. Magn Reson Imaging 21:1251-1261.

Granger C (1969) Investigating causal relations by econometric models and cross-spectral methods. Econometrica 37:424-438.

Granger C (1980) Testing for causality: a personal viewpoint. J Econ Dyn Control 2:329-352.

Griffiths TD, Buchel C, Frackowiak RS, Patterson RD (1998) Analysis of temporal structure in sound by the human brain. Nat Neurosci $1: 422-427$.

Griffiths TD, Kumar S, Warren JD, Stewart L, Stephan KE, Friston KJ (2007) Approaches to the cortical analysis of auditory objects. Hear Res 229:4653.

Guenther FH, Ghosh SS, Tourville JA (2006) Neural modeling and imaging of the cortical interactions underlying syllable production. Brain Lang 96:280-301.

Hackett TA, Stepniewska I, Kaas JH (1998) Subdivisions of auditory cortex and ipsilateral cortical connections of the parabelt auditory cortex in macaque monkeys. J Comp Neurol 394:475-495.

Hackett TA, Preuss TM, Kaas JH (2001) Architectonic indentification of the core region in auditory cortex of macaques, chimpanzees, and humans. J Comp Neurol 441:197-222.

Hickok G, Poeppel D (2004) Dorsal and ventral streams: a framework for understanding aspects of the functional anatomy of language. Cognition 92:67-99.

Hickok G, Poeppel D (2007) The cortical organization of speech processing. Nat Rev Neurosci 8:393-402.

Horwitz B (1990) Simulating functional interactions in the brain: a model for examining correlations between regional cerebral metabolic rates. Int J Biomed Comput 26:149-170.

Horwitz B (2003) The elusive concept of brain connectivity. NeuroImage 19:466-470

Howard MA, Volkov IO, Mirsky R, Garell PC, Noh MD, Granner M, Damasio H, Steinschneider M, Reale RA, Hind JE, Brugge JF (2000) Auditory cortex on the human posterior superior temporal gyrus. J Comp Neurol 416:79-92.

Kaas JH, Hackett TA (2000) Subdivisions of auditory cortex and processing streams in primates. Proc Natl Acad Sci USA 97:11793-11799.

Kumar S, Stephan KE, Warren JD, Friston KJ, Griffiths TD (2007) Hierarchical processing of auditory objects in humans. PLoS Comput Biol 3:e100.

Lehericy S, Ducros M, Van de Moortele PF, Francois C, Thivard L, Poupon C, Swindale N, Ugurbil K, Kim DS (2004) Diffusion tensor fiber tracking shows distinct corticostriatal circuits in humans. Ann Neurol 55:522-529.

Liegeois-Chauvel C, Musolino A, Chauvel P (1991) Localization of the primary auditory area in man. Brain 114:139-151

Liegeois-Chauvel C, Musolino A, Badier JM, Marquis P, Chauvel P (1994) Evoked potentials recorded from the auditory cortex in man: evaluation and topography of the middle latency components. Electroencephalogr Clin Neurophysiol 92:204-214.

McIntosh AR, Gonzales-Lima F (1994) Structural equation modeling and its application to network analysis in functional brain imaging. Hum Brain Mapp 2:2-22.

McIntosh AR, Grady CL, Ungerleider LG, Haxby JV, Rapoport SI, Horwitz B (1994) Network analysis of cortical visual pathways mapped with PET. J Neurosci 14:655-666.

Morel A, Kaas JH (1992) Subdivisions and connections of auditory cortex in owl monkeys. J Comp Neurol 318:27-63.

Morosan P, Rademacher J, Schleicher A, Amunts K, Schormann T, Zilles K (2001) Human primary auditory cortex: cytoarchitectonic subdivisions and mapping into a spatial reference system. NeuroImage 13:684-701. 
Pandya DN (1995) Anatomy of the auditory cortex. Rev Neurol (Paris) 151:486-494.

Parker GJ, Haroon HA, Wheeler-Kingshott CA (2003) A framework for a streamline-based probabilistic index of connectivity (PICo) using a structural interpretation of MRI diffusion measurements. J Magn Reson Imaging 18:242-254.

Rademacher J, Morosan P, Schormann T, Schleicher A, Werner C, Freund HJ, Zilles K (2001) Probabilistic mapping and volume measurement of human primary auditory cortex. NeuroImage 13:669-683.

Roebroeck A, Formisano E, Goebel R (2005) Mapping directed influence over the brain using Granger causality and fMRI. NeuroImage 25:230-242.

Romanski LM, Bates JF, Goldman-Rakic PS (1999a) Auditory belt and parabelt projections to the prefrontal cortex in the rhesus monkey. J Comp Neurol 403:141-157.

Romanski LM, Tian B, Fritz J, Mishkin M, Goldman-Rakic PS, Rauschecker JP (1999b) Dual streams of auditory afferents target multiple domains in the primate prefrontal cortex. Nat Neurosci 2:1131-1136.

Romanski LM, Tian B, Fritz J, Mishkin M, Goldman-Rakic PS, Rauschecker JP (2000) Reply to "What", "where" and "how" in auditory cortex. Nat Neurosci 3:966.

Scott SK, Blank CC, Rosen S, Wise RJ (2000) Identification of a pathway for intelligible speech in the left temporal lobe. Brain 123:2400-2406.

Sigalovsky IS, Fischl B, Melcher JR (2006) Mapping an intrinsic MR prop- erty of gray matter in auditory cortex of living humans: a possible marker for primary cortex and hemispheric differences. NeuroImage 32:1524-1537.

Talavage TM, Sereno MI, Melcher JR, Ledden PJ, Rosen BR, Dale AM (2004) Tonotopic organization in human auditory cortex revealed by progressions of frequency sensitivity. J Neurophysiol 91:1282-1296.

Tardif E, Clarke S (2001) Intrinsic connectivity of human auditory areas: a tracing study with Dil. Eur J Neurosci 13:1045-1050.

Tian B, Reser D, Durham A, Kustov A, Rauschecker JP (2001) Functional specialization in rhesus monkey auditory cortex. Science 292:290-293.

Upadhyay J, Ducros M, Knaus TA, Lindgren KA, Silver A, Tager-Flusberg H, Kim DS (2007) Function and connectivity in human primary auditory cortex: a combined fMRI and DTI study at 3 tesla. Cereb Cortex 17:2420-2432.

Warren JD, Zielinski BA, Green GG, Rauschecker JP, Griffiths TD (2002) Perception of sound-source motion by the human brain. Neuron 34:139-148.

Wilson MD (1988) The MRC psycholinguistic database: machine readable dictionary, version 2. Behav Res Methods Instrum Comput 20:6-11.

Zatorre RJ, Evans AC, Meyer E, Gjedde A (1992) Lateralization of phonetic and pitch discrimination in speech processing. Science 256:846-849.

Zimmer U, Macaluso E (2005) High binaural coherence determines successful sound localization and increased activity in posterior auditory areas. Neuron 47:893-905. 